\title{
RELAÇÃO PRELIMINAR DOS PEIXES DA ILHA DE SÃO LUIIS, MARANHÃO, BRASIL ${ }^{1}$
}

\author{
Ilídia da Ascenção Garrido Martins-Juras ${ }^{2}$ \\ Anastacio Afonso Juras ${ }^{2}$ \\ Naércio Aquino Menezes ${ }^{3}$
}

\begin{abstract}
This paper contains a preliminary list of the fishes caught monthly during the period between March, 1982 and April, 1986, in the littoral of the São Luis Island, and estuarine region including the localities Cururuca, Paciência, Cachorros and Ana Jansen, and the straits Coqueiro/Mosquitos. 132 species belonging to 94 genera and 56 families were obtained. Salinity ranged between 1.0 - 37.5\% oo at Cururuca and Paciência; 3.9 - 20.8\% oo at Ana Jansen, and 1.0 - 32.7\% $/ 00$ in the area including Mosquitos/Coqueiro and Cachorros. No correlation between salinity and distribution of species was obtained. Genyatremus luteus was the predominant species (21.4\%) followed by Mugil curema (11.6\%) and Arius herzbergii (11.2\%). Ariidae, Mugilidae, Sciaenidae and Engraulidae were the most representative families in number of individuals and species.
\end{abstract}

\section{INTRODUÇÃO}

A Ilha de Sáo Luís (MA) tem uma superfície da ordem de 90.500 hectares, estando inserida no Golfão Maranhense, formado pelas baías de São Marcos e São José. A região apresenta amplitudes de maré de cerca de sete metros, o que significa uma intensa invasão do continente pelas águas marinhas, cujo resultado é o aparecimento de largas faixas de sedimentos lamosos, colonizados por denso cinturão de mangues.

As águas da região são tipicamente estuarinas e resultantes da mistura das águas doces oriundas dos rios Pindaré e Mearim que desembocam na baía de São Marcos, e dos rios Itapecuru e Munim que desaguam na baía de São José.

No Estado do Maranhão, a pesca litorânea é praticada ainda de forma artesanal, constituindo-se em uma atividade de subsistência.

Este é um trabalho preliminar sobre a ictiofauna que ocorre no litoral da Ilha de São Luís, que tem por objetivo dar a conhecer a composição

1 Trabalho realizado com auxílio dos Convênios SUDEPE/UFMA e ALUMAR/UFMA.

2 Bolsista do CNPq.

3 Museu de Zoologia da Universidade de São Paulo. 
das espécies e fornecer subsídios sobre a distribuição das mesmas.

\section{METODOLOGIA}

Foram realizadas amostragens mensais em 4 diferentes localidades (número das estaçбes de coleta entre parênteses): estuários dos rios Cururuca (4) e Paciência (3), área de Mosquitos/Coqueiro e Cachorros (4) e Lagoa Ana Jansen (1) apresentadas na Figura 1, no período de março/82 a abril/86, utilizando-se redes de tapagem e espinhel.

Redes de tapagem, também denominadas "redes de igarapé", são aparelhos de pesca fixos, com extensão de aproximadamente 150 metros, medindo 4-5 metros de altura e com tamanho entrenós de $1-2 \mathrm{~cm}$; estas redes sáo dispostas na entrada do igarapé, sustentadas por varas de mangues, fixadas durante a baixamar, tendo a parte inferior fixa; na preamar a rede fica suspensa, sustentada pelas varas de mangue, sendo que a despesca é realizada na baixamar seguinte, totalizando um esforço de pesca de 12 horas. No espinhel, foram utilizados anzóis de diferentes tamanhos, tendo como isca peixe e camarão; o espinhel permaneceu na água por um período de 4 horas.

Os peixes capturados em cada local de amostragem foram acondicionados em sacos plásticos devidamente etiquetados, colocados em caixas de isopor contendo gelo, e transportados ao laboratório onde foi efetuada a separação e identificação das espécies. De cada espécie uma sub-amostra foi conservada em formol a $10 \%$ para identificação taxonômica. Esses peixes acham-se depositados no Museu de Zoologia da Universidade de São Paulo e no Departamento de Biologia da Universidade Federal do Maranhão.

Durante as coletas de peixes foram tomadas amostras de água para determinação da salinidade, efetuada em laboratório pelo método de MohrKnudsen.

\section{RESULTADOS}

Foram identificadas 132 espécies de peixes pertencentes a 56 famílias e 94 gêneros, de acordo com a lista abaixo, onde os nomes de classes e famílias seguem a ordenação proposta por Nelson (1984), acompanhados dos nomes vulgares regionais.

\section{I - CLASSE CHONDRICHTHYES}

Família Ginglimostomatidae

Ginglimostoma cirratum (Bonatore, 1788) - "cação arumaru"

Família Carcharhinidae

Rhizoprionodon lalandei (Valenciennes) - "figuinho" 


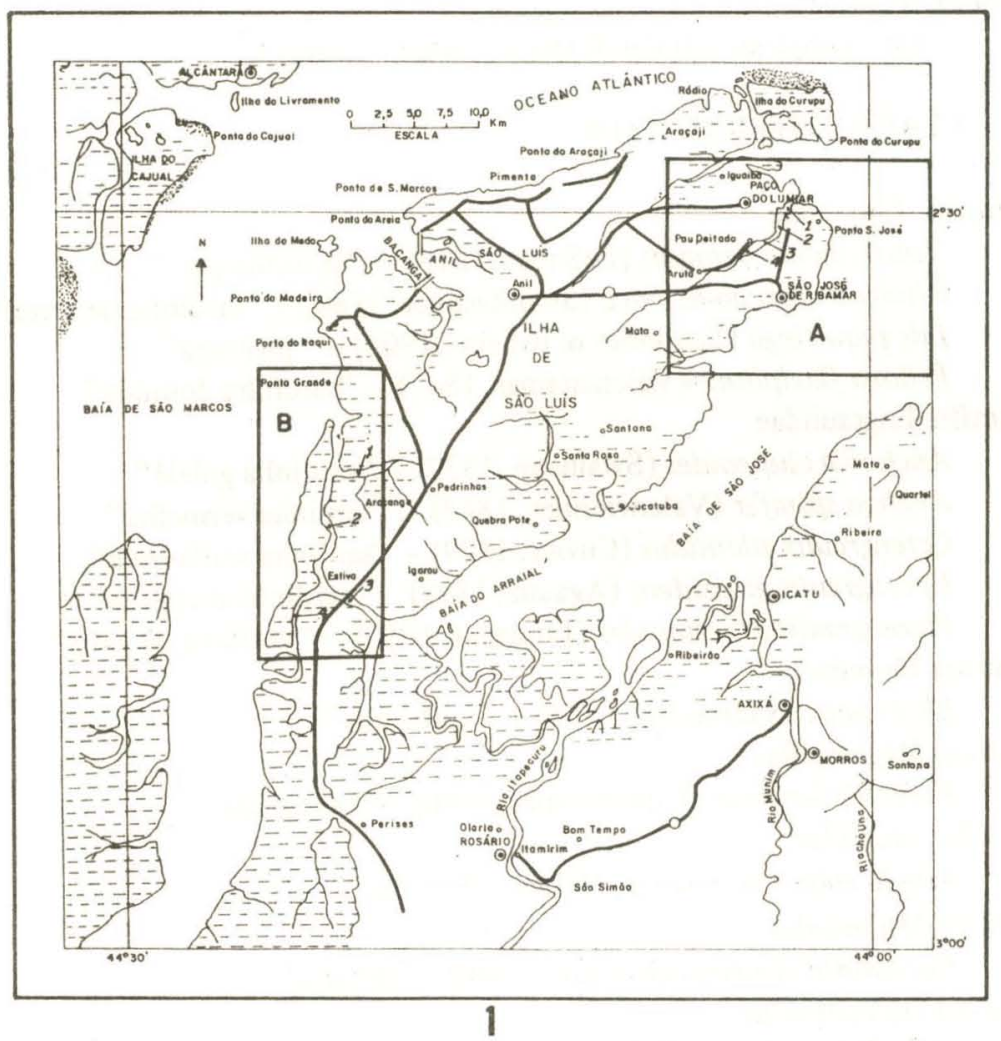

Fig. 1 - Mapa da Itha de São Luís mostrando as regiões onde foram efetuadas as coletas de peixes.

Rhizoprionodon porosus (Poey) - "figuinho"

Isogomphodon oxyrhynchus (Muller \& Henle, 1837) - "cação bicudo"

Carcarhinus porosus Ranzani - "junteiro"

Familia Shyrnidae

Sphyrna tudes (Valenciennes, 1822) - "cação rudela"

Sphyrna lewini (Cuvier, Grifith \& Muller) - "cação rudela"

Sphyma tiburo tiburo (Linnaeus) - "cação rudela"

Família Torpedinidae

Narcine brasiliensis (Olfers, 1831) - "arraia"

Famnlia Dasyatidae

Dasiatis guttata (Bloch, 1801) - "arraia-bicuda"

Dasiatis gejskesi (Boeseman, 1948) - "arraia-morcego"

Gymnura micrura (Bloch \& Schneider, 1801) - "arraia-baté"

Família Rhinopteridae

Rhinoptera bonasus (Mitchill, 181 5) - "raia"

Família Mobulidae

Mobula hyppostoma (Bancroft, 1831) - "jamanta" 
Familia Pristidae

Pristis perotteti (Muller \& Henle, 1840) - "serra"

\section{II - CLASSE OSTEICHTHYES}

Familia Clupeidae

Opisthonema oglinum (LeSueur, 1818) - "sardinha-peú"

Rhinosardinia amazonica (Steindachner, 1880) - "sardinha de serra"

Lile piquitinga (Schreiner \& Ribeiro, 1903) - "pititinga"

Pellona flavipinnis (Valenciennes, 1847) - "sardinha dourada"

Família Engraulidae

Anchovia clupeoides (Swainson, 1839) - "sardinha gulelê"

Anchoa spinifer (Valenciennes, 1848) - "sardinha vermelha"

Cetengraulis edentulus (Cuvier, 1829) - "sardinha verdadeira"

Lycengraulis grossidens (Agassiz, 1829) - "sardinha manjuba"

Pterengraulis atherinoides (Linnaeus, 1756) - "sardinha de gato"

Família Elopidae

Elops saurus (Linnaeus, 1766) - "uru barana"

Família Megalopidae

Tarpon atlanticus (Valenciennes, 1846) - "perapema"

Família Albulidae

Albula vulpes (Linnaeus, 1758) - "bicudo"

Família Muraenidae

Lycodontis funebris (Ranzani, 1840) - "moréia"

Família Ophichthidae

Ophichthus parilus (Richardson, 1844) - "jucutuca"

Família Synodontidae

Synodus foetens (Linnaeus, 1766) - "jacaré"

Família Serrasalmidae

Serrasalmus nattereri (Kner, 1860) - "piranha caju"

Familia Ariidae

Arius couma (Valenciennes, 1839) - "bagre catinga"

Arius herzbergii (Bloch, 1794) - "bagre guribu"

Arius quadriscutis (Valenciennes, 1840) - "cangatā"

Arius proops (Valenciennes, 1839) - "uritinga"'

Arius parkeri (Trail, 1832) - "gurijuba"

Arius grandicassis (Valenciennes, 1840) - "cambéu"

Arius rugispinnis (Valenciennes, 1840) - "jurupìranga"

Bagre bagre (Linnaeus, 1766) - "bagre bandeirado"

Cathorops spixii (Agassiz, 1829) - "bagrinho"

Cathorops sp. - "bagre uriacica"

Familia Auchenipteridae

Pseudauchenipterus nodosus (Bloch, 1749) - "papista"

Família Ageneiosidae

Ageneiosus sp. 
Familia Aspredinidae

Aspredinichthyes tibicen (Temminck, 1840) - "viola"

Aspredo aspredo (Linnaeus, 1758) - "viola"

Família Loricariidae

Hyppostomus cff. verres (Valenciennes, 1840) - "acari-bodó"

Familia Callichthyidae

Callichthys callichthys (Linnaeus, 1758) - "cascudo"

Familia Batrachoididae

Batrachoides surinamensis (Bloch \& Schneider, 1801) - "pacamão"

Thalassophryne nattereri (Steindachneri, 1976) - "niquim"

Familia Ogcocephalidae

Ogcocephalus verpertilio (Linnaeus, 1759) - "bacacuá"

Família Exocoetidae

Cypselurus melanurus (Valenciennes, 1846) - "voador"

Família Belonidae

Strongylura timucu (Walbaum, 1792) - "agulha"

Strongylura marina (Walbaum, 1792) - "agulha"

Família Atherinidae

Xenomelaniris brasiliensis (Quoy \& Gaimard, 1824) - “joão-duro"

Família Anablepidae

Anableps anableps (Linnaeus, 1759) - "tralhoto"

Anableps microlepis (Muller, 1844) - "tralhoto"

Família Scorpaenidae

Scorpaena plumieri (Bloch, 1789) - "mangangá"

Família Centropomidae

Centropomus parallelus (Poey, 1860) - "camurim branço"

Centropomus undecimalis (Bloch, 1792) - "camurim preto"

Família Serranidae

Diplectrum radiale (Quoy \& Gaimard, 1824) - "papa-terra"

Epinephelus itajara (Lichtenstein, 1822) - "mero"

Myctoperca bonasi (Poey, 1860) - "sirigado"

Polyprion americanus (Bloch \& Schneider, 1801) - "niquim mero"

Familia Gramistidae

Rypticus randalli (Courtenay, 1967) - "Peixe-sabđo"

Família Echeneidae

Echeneis naucrates (Linnaeus, 1758) - "rêmora"

Família Carangidae

Caranx hippos (Linnaeus, 1766) - "xaréu"

Caranx chrysus (Mitchill, 1815) - "xaréu"

Caranx latus (Agassiz, 1831) - "xaréu"

Chloroscombrus chrysurus (Linnaeus, 1766) - "favinha"

Hemicaranx amblyrhynchus (Cuvier, 1833) - "xixarro"

Oligoplites palometa (Cuvier, 1833) - "tibiro amarelo"

Oligoplites saurus (Bloch \& Schneider, 1801) - "tibiro branco"

Selene vomer (Linnaeus, 1758) - "peixe galo" 
Trachinotus falcatus (Linnaeus, 1758) - "pampo"

Trachinotus carolinus (Linnaeus, 1766) - "pampo"

Trachinotus cayannensis (Cuvier, 1833) - "pampo"

Família Lutjanidae

Lutjarus synagris (Linnaeus, 1758) - "carapitanga"

Lutjanus jocu (B1nch \& Schneider, 1801) - "carapitanga"

Família Lobotidae

Lobotes surinamensis (Bloch, 1790) - "crauaçu"

Familia Gerreidae

Diapterus olisthostomus (Goode \& Bean, 1882) - "peixe-prata"

Diapterus rhombeus (Cuvier, 1829) - "peixe-prata"

Eucinostomus argenteus (Baird \& Girard, 1845) - "escrivão"

Eucinostomus gula (Cuvier, 1830) - "escrivão"

Eucinostomus melanopterus (Bleeker, 1863) - "escrivão"

Eugerres brasilianus (Cuvier, 1830) - "carapitanga"

Família Pomadasyidae

Conodon nobilis (Linnaeus, 1758) - "jiqui-listrado"

Genyatremus luteus (Bloch, 1795) - "peixe-pedra"

Orthopristes ruber (Cuvier, 1830) - "corcoroca"

Pomadasys corvaeniformes (Steindachner, 1869) - "jiquiri-branco"

Família Sparidae

Archosargus probatocephalus (Walbaum, 1792) - "sorgo"

Familia Sciaenidae

Cynoscion leiarchus (Cuvier, 1830) - "curvitinga"

Cynoscion microlepidotus (Cuvier, 1830) - "corvina uçu"

Cynoscion steindachneri (Jordan, 1889) - "juruapara"

Cynoscion acoupa (Lacépède, 1802) - "pescada vermelha"

Macrodon ancylodon (Bloch \& Schneider, 1801) - "corvina-gó"

Menticirrhus americanus (Linnaeus, 1758) - "boca-de-rato"

Micropogonias furnieri (Desmarest, 1823) - "cururuca"

Stellifer stellifer (Bloch, 1790) - "cabeçudo vermelho"

Stellifer rastrifer (Jordan, 1889) - "cabeçudo vermelho"

Stellifer brasiliensis (Schultz, 1945) - "cabeçudo"

Atellifer naso (Jordan, 1889) - "cabeçudo preto"

Bairdiella ronchus (Cuvier, 1830) - "cororoca"

Isopisthus parvipinnis (Cuvier, 1830) - "curvitinga"

Nebris microps (Cuvier, 1830) - "olho-de-moça"

Hamilia Ephippidae

Chaetodipterus faber (Broussonet, 1782) - "paru-preto"

Família Pomacanthidae

Pomacanthus paru (Bloch, 1787) - "frade"

ramília Mugilidae

Mugil curema (Valenciennes, 1830) - "tainha sajuba"

Mugil incilis (Hancock, 1830) - "tainha urixoca"

Mugil trochodon (Poey, 1876) - "tainha" 
Mugil gaimardianus (Desmarest, 1831) - "tainha pitiu"

Mugil liza (Valenciennes, 1836) - "tainha curima"

Família Polynemidae

Polydactylus oligodon (Gunther, 1860) - "barbudo"

Polydactylus virginicus (Linnaeus, 1758) - "barbudo"

Família Eleotridae

Guavina guavina (Valenciennes, 1837) - "muré"

Familia Gobiidae|

Gobionellus shufeldti (Jordan \& Eigemann, 1886) - "muré"

Bathygobius soporator (Valenciennes, 1837) - "muré"

Gobionellus oceanicus (Pallas, 1770) - "mure"

Familia Scombridae

Scomberomorus regalis (Bloch, 1793) - "serra"

Familia Stromateidae

Peprilus paru (Linnaeus, 1758) - "canguiro"

Família Trichiuridae

Trichiurus lepturus (Linnaeus, 1758) - "guaravira"

Família Bothidae

Paralichthys sp. - "linguado"

Etropus crossotus (Jordan \& Gilbert, 1882) - "solha urumaçara"

Citarichthys spilopterus (Gunther, 1862) - "solha urumaçara"

Família Soleidae

Achirus achirus (Linnaeus, 1758) - "solha verdadeira"

Trinectes cff. paulistanus - "solha verdadeira"

Familia Cynoglossidae

Symphurus plagusia (Bloch \& Schneider, 1801) - "linguado"

Família Monacanthidae

Alutera monoceros (Linnaeus, 1758) - "cângulo"

Famflia Tetraodontidae

Colomesus psittacus (Bloch \& Schneider, 1801) - "baiacu-açu"

Lagocephalus laevigatus (Linnaeus, 1766) - "baiacu-guará"

Sphoeroides greeleyi (Gilbert, 1900) - "baiacu-areia"

Sphoeroides testudineus (Linnaeus, 1758) - "baiacu-pininga"

Família Diodontidae

Chilomycterus antillarum (Jordan \& Rutter, 1897) - "baiacu-de-espinho"

\section{DISCUSSÃO E CONCLUSÖES}

Poucos são os trabalhos referentes à ictiofauna desta regiao. Entre eles há um levantamento nas Reentrâncias Maranhenses (SUDEPE, 1976), onde foram assinaladas 43 espécies; na plataforma continental maranhense foram registradas 52 espécies (SUDENE, 1976). Na Itha de São Luís foi realizado um levantamento dos peixes comercializados no estuário do rio Cururuca 
(SUDAM, 1983) onde foram registradas 50 espécies.

No período abrangido por este estudo, a salinidade variou de 1,0 a $37,5 \%$ nos estuários dos rios Cururuca e Paciência, de 1,0 a $32,7 \%$ oo na área de Mosquitos/Coqueiro e Cachorros e de 3,9 a 20,8\% oo na Lagoa Ana Jansen. Não se verificou qualquer tipo de associação entre espécies ou grupos de espécies em função da variação da salinidade.

Tabela 1 - Dados sobre a salinidade das águas, por estação de coleta no Estuário do Rio Cururuca (A) e Estreitos do Coqueiro e Mosquitos e Rio dos Cachorros (B).

\begin{tabular}{|c|c|c|c|c|c|c|c|c|c|}
\hline \multirow{2}{*}{ Ano } & \multirow{2}{*}{ Meses } & \multicolumn{4}{|c|}{ A } & \multicolumn{4}{|c|}{ B } \\
\hline & & I & II & III & IV & I & II & III & IV \\
\hline \multirow{8}{*}{ 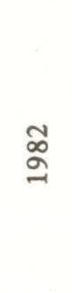 } & maio & - & - & - & - & - & - & - & - \\
\hline & junho & - & - & - & - & 23,3 & 22,1 & 22,8 & - \\
\hline & julho & - & 32,8 & 28,6 & 23,0 & 24,6 & 22,9 & 22,9 & 22,9 \\
\hline & agosto & 30,5 & 30,3 & 28,4 & 21,2 & 26,5 & 26,0 & 26,2 & 28,0 \\
\hline & setembro & 36,1 & 37,2 & 36,1 & 28,5 & 29,0 & 28,0 & 28,0 & 28,0 \\
\hline & outubro & 36,4 & 37,0 & 36,0 & 30,6 & 31,0 & 30,7 & 30,7 & 30,0 \\
\hline & novembro & 36,3 & 32,0 & 31,0 & 30,8 & 32,6 & 32,0 & 32,0 & 32,6 \\
\hline & dezembro & 37,3 & 35,8 & 35,8 & 34,6 & 31,0 & 31,2 & 31,0 & 31,0 \\
\hline \multirow{5}{*}{$\begin{array}{l}\infty \\
\infty \\
\approx\end{array}$} & janeiro & 37,5 & 36,5 & 37,0 & 37,0 & 32,3 & 31,9 & 32,0 & 32,5 \\
\hline & fevereiró & 34,0 & 32,0 & 30,5 & 28,0 & 30,4 & 28,2 & 26,9 & 26,0 \\
\hline & março & 34,0 & 34,0 & 34,0 & 28,0 & 25,5 & 22,9 & 21,5 & 30,0 \\
\hline & abril & 33,4 & 32,5 & 32,3 & 29,0 & 24,0 & 23,9 & 24,8 & 24,1 \\
\hline & maio & - & - & - & - & 24,9 & 22,9 & 22,0 & 21,0 \\
\hline
\end{tabular}

Dos peixes capturados observou-se predominância numérica de Genyatremus luteus $(21,4 \%)$, Mugil curema $(11,6 \%)$ e Arius herz bergii $(11,2 \%)$. Pseudauchenipterus nodosus, Hyppostomus cf. verres, Aspredo aspredo, Ageneiosus sp., Pellona flavipinnis e Serrasalmus nattereri ocorreram apenas na estação chuvosa, onde os valores de salinidade são baixos. Genyatremus luteus, Arius herzbergii, Colomesus psittacus e Mugil curema ocorreram em todas as épocas do ano, evidenciando uma ampla distribuição e uma grande tolerância às variaçбes da salinidade.

Estudos sobre composição e distribuição da fauna estuarina de peixes na região nordeste revelaram uma predominância de espécies marinhas sobre as de água doce (Menezes \& Menezes, 1968; Oliveira, 1976, 1980), corroborando os resultados obtidos neste estudo.

Embora os dados apresentados neste trabalho sejam preliminares, foi possível verificar que na regiao estudada houve predominância de representantes das familias Ariidae, Mugilidae, Sciaenidae e Engraulidae, tanto em número de indivíduos como de espécies. É bem conhecido o fato de espécies dessas familias procurarem águas estuarinas para reprodução ejou crescimen- 
to. Na lagoa dos Patos (Rio Grande do Sul), representantes de Sciaenidae, Ariidae, Engraulidae e Mugilidae estiveram entre as espécies mais abundantes nos diversos tipos de arrastos realizados (Chao, et al., 1982). Weiss (1981) encontrou na mesma lagoa dos Patos predominância de larvas das famílias Engraulidae, Clupeidae e Sciaenidae. Os dados do presente trabalho confirmam a tendência de predomínio de espécies destas famílias, embora no caso constituam, de uma maneira geral, espécies distintas daquelas encontradas no sudeste e sul do Brasil.

\section{REFERÊNCIAS}

Chao, L.N., Pereira, L.E. Vieira, J.P. Bemvenuti, M.A. \& Cunha, L.P.R. 1981. Relação preliminar dos peixes estuarinos e marinhos da lagoa dos Patos e região costeira adjacente, Rio Grande do Sul, Brasil. Attântica, Rio Grande, 5: 67-75.

Menezes, R.S. de \& Menezes, M.F. de 1968. Estudo preliminar sobre a flora e a fauna de águas estuarinas do Estado do Ceará. Arq. Est. Biol. Mar. Univ. Fed. Ceará, 8 (11) $101-106$.

Nelson, J.S. 1984. Fishes of the World. John Wiley \& Sons, XIII, 416 pp.

Oliveira A.M.E. de 1976. Composição e distribuição da ictiofauna nas águas estuarinas do Rio Jaguaribe (Ceará, Brasil). Arq. Ciênc. Mar, 16 (1): 9-18.

Oliveira, A.M.E. de 1980. Distribution of estuarine fishes in Northeastern Brazil in relation to water salinity. Memorias del Seminario sobre el Estudio Científico e Impacto Humano en el Ecosistema de Manglares. UNESCO, 94-101.

Sudam, 1983. Superintendência do Desenvolvimento da Amazônia. Caracterização Am. biental e Prospeç̧ão Pesqueira do Estuário do Rio Cururuca, Maranhão. Belém, $141 \mathrm{pp}$.

Sudene, 1976. Superintendência do Desenvolvimento do Nordeste. Pesquisas dos Recursos Pesqueiros Maranhenses, Série Estudos de Pesca, nọ 6, 76 pp.

Sudepe, 1976. Superindentência do Desenvolvimento da Pesca. Prospecção dos Recursos Pesqueiros das Reentrâncias Maranhenses. Governo do Maranhão, 124 pp.

Weiss, G. 1981. Ictioplancton del estuario de Lago dos Patos, Brasil. Tesis de Doutorado, Universidad Nacional de la Plata. Faculdad de Ciencias Naturales y Museo, 164 pp. 